\title{
A Rare Agent of Urinary Tract Infection: Chryseobacterium indologenes
}

\author{
Nadir Bir İrar Yolu Enfeksiyonu Etkeni: Chryseobacterium indologenes
}

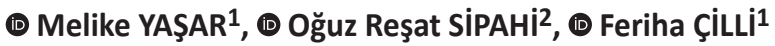 \\ 1 Ege University Faculty of Medicine, Department of Medical Microbiology, Izmir, Turkey \\ ${ }^{2}$ Ege University Faculty of Medicine, Department of Infectious Diseases and Clinical Microbiology, Izmir, Turkey
}

Keywords: Complicated urinary tract infection, rifampin, lovofloxacin, imipenem, meropenem

Anahtar Kelimeler: Komplike üriner sistem enfeksiyonu, rifampin, levofloksasin, imipenem, meropenem

\section{Dear Editor,}

Members of the genus Chryseobacterium, formerly known as Flavobacterium, are not human flora members despite being commonly found in nature. They are a potential source of infection due to their ability to survive in chlorinated municipal water supplies and their presence in hospital water systems and tap water.

Chryseobacterium indologenes is an immobile, catalasepositive, oxidase-positive, indole-positive, Gram-negative non-fermenting bacillus ${ }^{[1]}$. As this bacterium is not frequently detected in clinical specimens, its clinical significance has yet to be determined ${ }^{[2]}$. It is rarely pathogenic in humans and most of the reported cases are hospital-acquired infections. It usually causes infection in immunocompromised patients and those with permanent catheters. It has been reported as the causative agent in cases of bacteremia, peritonitis, pneumonia, empyema, pyelonephritis, cystitis, meningitis, and central venous catheterrelated infections ${ }^{[1-9]}$. Herein, we present a patient who had a urinary catheter, was scheduled for a urological procedure, and whose urine culture yielded $C$. indologenes.

A 66-year-old woman had presented to another center one month earlier and inserted an indwelling urinary catheter due to complaints of dysuria and subsequent inability to urinate. Cystoscopy was performed during follow-up but evaluation could not be completed due to pain. Therefore, the patient was recommended cystoscopy under anesthesia. Urine culture performed before the planned urologic procedure yielded $10^{5}$ colony-forming units (CFU)/ml of panresistant Pseudomonas aeruginosa, upon which she was admitted to the the Infectious Diseases and Clinical Microbiology Department for testing and treatment. The patient reported itching and pain at the entry point of the indwelling Foley catheter. She had no other complaints when questioned and physical examination revealed no other pathological findings. Her medical history showed that she had undergone mitral valve replacement twice. Laboratory tests revealed red blood cell count: 3,690,000/ $\mathrm{mm}^{3}$, hemoglobin level: $12.6 \mathrm{~g} / \mathrm{dl}$, hematocrit: $36.2 \%$, white blood cell (WBC) count: 5130/mm ${ }^{3}$, CRP: $0.44 \mathrm{mg} / \mathrm{dl}$, aspartate aminotransferase: $27 \mathrm{U} / \mathrm{l}$, alanine aminotransferase: $17 \mathrm{U} / \mathrm{I}$, urea: $42 \mathrm{mg} / \mathrm{dl}$, and creatinine: $0.74 \mathrm{mg} / \mathrm{dl}$. At admission, the patient was started on parenteral meropenem $6 \mathrm{~g} /$ day and imipenem $2 \mathrm{~g} /$ day, and peroral rifampicin $600 \mathrm{mg} /$ day for $P$. aeruginosa. Urine cultures obtained on day of admission (direct microscopy: 50 leukocytes $/ \mathrm{mm}^{3}$ ) and the following day, when she developed a fever (direct count: 10 leukocytes $/ \mathrm{mm}^{3}$ ) yielded $10^{5} \mathrm{cfu} / \mathrm{ml} \mathrm{C}$. indologenes. Blood culture obtained during the fever episode negative. Following this, two more consecutive catheter urine samples were taken from the patient and sent to the Microbiology Laboratory for culture. Direct microscopic examination of the samples revealed $50 \mathrm{WBC} / \mathrm{mm}^{3}$ and 3-4 red blood cells per field. Parenteral levofloxacin $1 \mathrm{~g} /$ day was added

Cite this article as: Yaşar M, Sipahi OR, Çilli F. A Rare Agent of Urinary Tract Infection: Chryseobacterium indologenes. Mediterr J Infect Microb Antimicrob. $2019 ; 8: 15$. 
to the patient's antibiotic therapy because susceptibility testing indicated resistance to imipenem and meropenem. Follow-up urine cultures sent four and seven days after the initiation of levofloxacin were negative. On day six of clinical follow-up, the patient's fever relapsed $\left(38{ }^{\circ} \mathrm{C}\right)$ and blood cultures were repeated. On day seven of inpatient treatment, while under antibiotherapy, she had a widespread rash and fever. Her CRP level increased to $10.93 \mathrm{mg} / \mathrm{dl}$, another blood culture was performed. Dermatology department was consulted, and it was considered to be a drug reaction. Upon negative blood and urine cultures at the end of treatment, antibiotic therapy was discontinued after 10 days (imipenem and meropenem for 10 days, rifampicin for 11 days, levofloxacin for 9 days) and the patient was discharged. There was no relapse or reinfection at one-month follow-up.

In addition to direct microscobic examination (Fast Read $102^{\circ}$, Biosigma, Italy), urine samples from the patient's catheter were cultured by inoculating 5\% sheep blood agar (SBA) and eosin methylene-blue agar (BioMerieux, France). Blood samples taken from the patient were inoculated into aerobic and anaerobic blood culture bottles and loaded into a BacT/ALERT (BioMerieux, France) automated hemoculture system. Colonies producing yellow pigment were observed in the SBA urine culture after 24-48 hours of incubation in an aerobic atmosphere in $36{ }^{\circ} \mathrm{C}$ incubator. These colonies were identified as $C$. indologenes using a MALDI-TOF MS (Vitek MS) (BioMerieux, France).

The gradient test method was used to investigate the antimicrobial susceptibility pattern of the $C$. indologenes isolates. To do this, a bacterial suspension with a McFarland turbidity of 0.5 was prepared from the colonies and passaged onto Mueller-Hinton agar (MHA) (BioMerieux, France). The minimum inhibitory concentration (MIC) values of the antimicrobials tested were determined using E-test strips (BioMerieux, France). Minimum inhibitory concentration was $16 \mathrm{mg} / \mu \mathrm{l}$ (resistant) for piperacillin-tazobactam, $0.5 \mathrm{mg} /$ $\mu \mathrm{l}$ for ciprofloxacin (susceptible), $2 \mathrm{mg} / \mu \mathrm{l}$ for ceftazidime (susceptible), and $>32 \mathrm{mg} / \mu \mathrm{l}$ for imipenem and meropenem (resistant). Susceptibility results were evaluated using EUCAST FK/FD (species-independent) breakpoint values. Minimum inhibitory concentration values for rifampicin, fosfomycin, vancomycin, and trimethoprim-sulfamethoxazole were determined as $0.25 \mathrm{mg} / \mu \mathrm{l}, 1024 \mathrm{mg} / \mu \mathrm{l}, 32 \mathrm{mg} / \mu \mathrm{l}$, and 0.064 $\mathrm{mg} / \mu \mathrm{l}$, respectively. Since there were no breakpoint values for rifampicin, fosfomycin, vancomycin, and trimethoprimsulfamethoxazole in the EUCAST FK/FD table for reference, only MIC values were reported to the ward ${ }^{[10]}$.

Although C. indologenes is common in soil, water, plants, and food, it is rarely pathogenic in humans ${ }^{[3]}$. Its clinical significance has increased in recent years due to its ability to cause severe infections in patients who undergo invasive permanent interventions, those with underlying disease, immunocompromised, and newborns ${ }^{[1,5]}$.

C. indologenes may survive in chlorinated water and its presence in hospital environments, water systems, and wet surfaces poses a potential source of infection. Patients may be colonized from contaminated medical devices involving fluids, such as respirator masks, intubation tubes, incubators, and refrigerators/freezers ${ }^{[3]}$. Similarly, Bayraktar et al. ${ }^{[5]}$ demonstrated that bacterial infection was caused by contaminated distilled water in a hospitalized patient in whom $C$. indologenes was isolated in blood culture. Cases of bacteremia, meningitis, and tracheobronchitis associated with C. indologenes have been reported previously in Turkey, but no urinary tract infections ${ }^{[1,3,6-8]}$.

Chryseobacterium spp. are known to be resistant to aminoglycosides, tetracyclines, chloramphenicol, erythromycin, clindamycin, and teicoplanin. The most effective antimicrobials against these microorganisms are fluoroquinolones (garenoxacin, gatifloxacin, levofloxacin) and rifampicin. Trimethoprimsulfamethoxazole, ciprofloxacin, and piperacillin-tazobactam are other antimicrobial agents with fair antibacterial activity. Vancomycin shows poor potential ${ }^{[9]}$. The resistance profile of the strain isolated from our patient was similar to that of other $C$. indologenes strains reported in the literature. The patient was initially treated with imipenem, meropenem, and rifampicin for panresistant $P$. aeruginosa, after which levofloxacin was added to the regimen since Chryseobacterium spp. can be metallo- $B$ lactamase producers and the strain was found to be resistant to imipenem and meropenem.

In conclusion, bacteriological examination is important in patients who are immunocompromised, have underlying disease, and/or have prolonged use of invasive devices. These factors may lead to opportunistic infection by $C$. indologenes, albeit rarely. There is no optimal treatment regimen for Chryseobacterium spp. infections. Therefore, antimicrobial therapy should be based on MIC values of antimicrobial agents by correctly applied susceptibility tests ${ }^{[9]}$. To the best of our knowledge, this is the first reported case of $C$. indologenes-associated urinary tract infection in Turkey.

\section{Ethics}

Informed Consent: Consent form was filled out by the reported case.

Peer-review: Externally and internally peer-reviewed.

\section{Authorship Contributions}

Concept: O.R.S., F.Ç., Design: O.R.S., F.Ç., Data Collection or Processing: M.Y., Analysis or Interpretation: O.R.S., F.Ç., Literature Search: M.Y., Writing: O.R.S., F.Ç., M.Y. 
Conflict of Interest: No conflict of interest was declared by the authors.

Financial Disclosure: The authors declared that this study received no financial support.

\section{References}

1. Ceylan A, Güdücüoğlu H, Akbayram S, Bektaş A, Berktaş M. Sepsis Caused by Chryseobacterium indologenes in a Patient with Hydrocephalus. Mikrobiyol Bul. 2011;45:735-40.

2. Lin YT, Jeng YY, Lin ML, Yu KW, Wang FD, Liu CY. Clinical and Microbiological Characteristics of Chryseobacterium indologenes Bacteremia. J Microbiol Immunol Infect. 2010;43:498-505.

3. Bhuyar G, Jain $S$, Shah $H$, Mehta VK. Urinary tract infection by Chryseobacterium indologenes. Indian J Med Microbiol. 2012;3:370-2.

4. Mirza HC, Tuncer Ö, Ölmez S, Şener B, Tuğcu GD, Özçelik U, Gürsoy NC, Otlu B, Büyükçam A, Kara A, Sancak B.Clinical Strains of Chryseobacterium and Elizabethkingia spp. Isolated from Pediatric Patients in a University Hospital: Performance of MALDI-TOF MS-Based Identification, Antimicrobial Susceptibilities, and Baseline Patient Characteristics. Microb Drug Resist. 2018; 24(6):816-821. Available from: https://www.liebertpub. com/doi/10.1089/mdr.2017.0206
5. Bayraktar MR, Aktas E, Ersoy Y, Cicek A, Durmaz R. Postoperative Chryseobacterium indologenes Bloodstream Infection Caused by Contamination of Distillate Water. Infect Control Hosp Epidemiol 2007;28:368-9.

6. Mutcali SI, Yemisen M, Soylu H, Balkan II, Mete B, Saltoglu N. Recurrent port infection due to Chryseobacterium indologenes. Eurasian J Emerg Med. 2013;45:60-1.

7. Teke $\mathrm{TA}, \mathrm{Oz}$ FN, Metin O, Bayhan Gl, Aydin ZGG, Oguz M, Tanir G. Chryseobacterium indologenes Septicemia in an Infant Case Rep Infect Dis. 2014;2014:270521. Available from: https://www.hindawi.com/journals/ criid/2014/270521/

8. Akay M, Gunduz E, Gulbas Z. Catheter-related bacteremia due to Chryseobacterium indologenes in a bone marrow transplant recipient. Bone Marrow Transplant. 2006;37:435-6.

9. Kirby JT, Sader HS, Walsh TR, Jones RN. Antimicrobial susceptibility and epidemiology of a worldwide collection of Chryseobacterium spp: report from the SENTRY Antimicrobial Surveillance Program (1997-2001). J Clin Microbiol 2004;42:445-8.

10. The European Committee on Antimicrobial Susceptibility Testing. Breakpoint tables for interpretation of MICs and zone diameters. Version 8.1, 2018. Available from: http://www.eucast.org 